\title{
Exercise opens a temporal window for enhanced cognitive improvement from subsequent physical activity
}

\author{
Christopher W. Butler, ${ }^{1}$ Ashley A. Keiser, ${ }^{1}$ Janine L. Kwapis, ${ }^{2}$ Nicole C. Berchtold, ${ }^{1}$ \\ Vanessa L. Wall, ${ }^{1}$ Marcelo A. Wood, ${ }^{1}$ and Carl W. Cotman ${ }^{1}$ \\ ${ }^{1}$ Department of Neurobiology and Behavior, University of California Irvine, Irvine, California 92617, USA; ${ }^{2}$ Department of Biology, \\ Center for Molecular Investigation of Neurological Disorders, Pennsylvania State University, University Park, Pennsylvania 16802, USA
}

\begin{abstract}
The beneficial effects of exercise on cognition are well established; however specific exercise parameters regarding the frequency and duration of physical activity that provide optimal cognitive health have not been well defined. Here, we explore the effects of the duration of exercise and sedentary periods on long-term object location memory (OLM) in mice. We use a weak object location training paradigm that is subthreshold for long-term memory formation in sedentary controls, and demonstrate that exercise enables long-term memories to form. We show that 14- and 21-d of running wheel access enables mice to discriminate between familiar and novel object locations after a $24 \mathrm{~h}$ delay, while 2- or 7-d running wheel access provides insufficient exercise for such memory enhancement using the subthreshold learning paradigm. After 14- and 21-d of wheel running, exercise-induced cognitive enhancement then decays back to baseline performance following 3-d of sedentary activity. However, exercise-induced cognitive enhancement can be reactivated by an additional period of just $2 \mathrm{~d}$ exercise, previously shown to be insufficient to induce cognitive enhancement on its own. The reactivating period of exercise is capable of enhancing memory after three- or seven-sedentary days, but not 14-d. These data suggest a type of "molecular memory" for the exercise stimulus, in that once exercise duration reaches a certain threshold, it establishes a temporal window during which subsequent low-level exercise can capitalize on the neurobiological adaptations induced by the initial period of exercise, enabling it to maintain the benefits on cognitive function. These findings provide new information that may help to guide future clinical studies in exercise.
\end{abstract}

A growing body of evidence arising from studies of both animal models and human subjects supports the beneficial effects of exercise on general health (Warburton et al. 2006; Nagamatsu et al. 2014). These benefits appear to demonstrate a dose-response relationship, as total physical activity volume shows an inverse linear relationship to all-cause mortality in humans (Lee and Skerrett 2001). Accordingly, the World Health Organization recommends adults aged 18-64 experience at least 150 min of moderate-intensity aerobic physical activity per week (Haskell et al. 2007; WHO 2010). More specifically, physical activity is associated with neurobiological benefits, including improved cognition, a reduction in the risk of developing a neurodegenerative disease, and attenuation of age-related cognitive decline (Cotman and Berchtold 2002; Gomez-Pinilla and Hillman 2013; Nagamatsu et al. 2014). However, much less is known of the minimal frequency or session duration of physical activity required to maintain such cognitive benefits (Alderman and Olson 2018). Indeed, exercise patterns in humans are less rigid than exercise regimens used in animal studies, and generally do not follow a consistent daily routine (Lee and Skerrett 2001).

A number of studies have examined the effects of exercise frequency on cognitive improvement in rodent models, by varying the duration of exercise and sedentary periods that rodents experience. Rodents display robust exercise-induced cognitive enhancement as measured by a wide variety of behavioral tests (Vivar et al. 2013). This cognitive enhancement has also been shown to eventually decay following a return to sedentary activity, as dem-

\section{Corresponding author: cwbutler@uci.edu}

Article is online at http://www.learnmem.org/cgi/doi/10.1101//m.050278.119. onstrated using the passive avoidance test (Radak et al. 2006), and radial arm water maze (Berchtold et al. 2010). One molecular correlate of this cognitive enhancement has been identified in the form of increased hippocampal brain-derived neurotrophic factor (BDNF) expression in rats and mice with access to running wheels (Neeper et al. 1996; Berchtold et al. 2005, 2010). Exercise on alternating days has been shown to be as effective in elevating hippocampal BDNF levels as daily exercise (Berchtold et al. 2005), and hippocampal BDNF levels remain elevated for $2 \mathrm{wk}$ following the complete cessation of exercise, returning to baseline levels after 3-4 wk in mice (Berchtold et al. 2010), and $14 \mathrm{~d}$ in rats (Berchtold et al. 2005). Interestingly, a subsequent reactivating period of exercise has been shown to return hippocampal BDNF levels back to levels that previously required 2 wk of exercise for induction in rats, although the effect on cognition was not examined (Berchtold et al. 2005). These data suggest that the initial exercise period primes the hippocampus so that subsequent low-level physical activity can induce rapid BDNF expression.

In this study, we investigate the effects of varying exercise frequency and duration on long-term object location memory, using a weak object location memory (OLM) training paradigm that is subthreshold for memory formation in sedentary controls. We have previously demonstrated that exercise enables learning in

2019 Butler et al. This article is distributed exclusively by Cold Spring Harbor Laboratory Press for the first 12 months after the full-issue publication date (see http://learnmem.cshlp.org/site/misc/terms.xhtml). After 12 months, it is available under a Creative Commons License (AttributionNonCommercial 4.0 International), as described at http://creativecommons. org/licenses/by-nc/4.0/. 
these normally subthreshold conditions, and that this learning is mediated by hippocampal BDNF (Intlekofer et al. 2013). We subject mice to differing exercise regimes that consist of varying duration of running wheel access, and standard sedentary cage housing, and determine the time course of decay of OLM caused by a return to sedentary activity. We also examine the effect on OLM of an additional period of reactivating exercise just prior to behavioral testing, to see if the previously discovered molecular memory for exercise-induced BDNF expression has an effect on long-term OLM.

\section{Results}

\section{Exercise enables mice to learn following subthreshold OLM training}

To investigate the effects of the quantity and frequency of different periods of exercise and sedentary activity on cognition in mice, we first established the degree to which different quantities of exercise can benefit mouse OLM (Fig. 1). Mice were given access to a running wheel for either $2 \mathrm{~d}$, or 1,2 , or $3 \mathrm{wk}$ prior to OLM training. Voluntary wheel-running was chosen because it allows mice to choose how much to run, therefore avoiding confounding variables associated with the stress of forced exercise, and is easily quantifiable (Cotman and Berchtold 2002). To test for memory enhancement, we used a subthreshold training time (3 min), which has been previously shown not to result in long-term OLM in sedentary wild-type mice, but can lead to longterm memory in mice that have undergone exercise (McQuown et al. 2011; Intlekofer et al. 2013; Vogel-Ciernia and Wood 2014). A sedentary group of mice was also included that received no access to running wheels for the duration of the experiment. Mice were handled and habituated to the OLM chamber as described prior to OLM training, regardless of individual exercise group (Fig. 1A).

While in running wheel cages, exercise was recorded automatically. The mean total distance covered by each group was proportional to the time spent in the running wheel cages (Fig. $1 \mathrm{~B} ; 2 \mathrm{~d}: M$

$=11,571$ meters, $S D=3157 \mathrm{~m}$; 1 wk: $M=32,567 \mathrm{~m}, S D=7847 \mathrm{~m}$; 2 wk: $M=101,557 \mathrm{~m}, S D=49,572 \mathrm{~m} ; 3$ wk: $M=129,786 \mathrm{~m}, S D=$ $\left.37,669 \mathrm{~m} ; F_{(3,28)}=29.23, P<0.0001\right)$, with mice receiving $3 \mathrm{wk}$ of running wheel exposure exercising the most. Mice from each group then received OLM training the day after the last day of running wheel access. Each group of mice displayed a similar total object exploration time during training, indicating no effect of exercise treatment on object exploration (Fig. 1C, $F_{(4,45)}=0.6179$, $P=0.6520, \mathrm{~ns})$. There was also no significant difference in the DI for the objects (Fig. $1 \mathrm{D}, F_{(4,45)}=1.464, P=0.2291$ ), indicating no

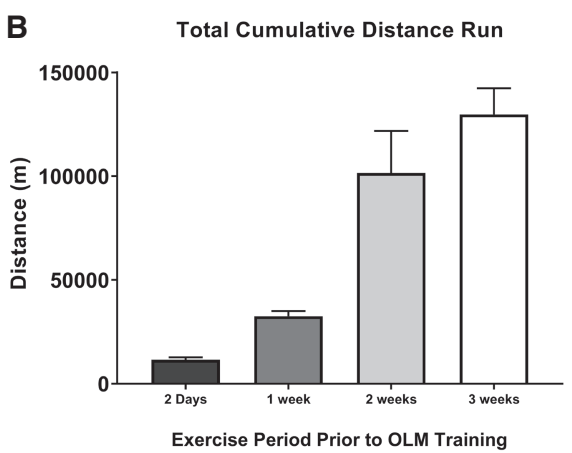

C Total Object Exploration During OLM Training

D

Object Preference During OLM Training

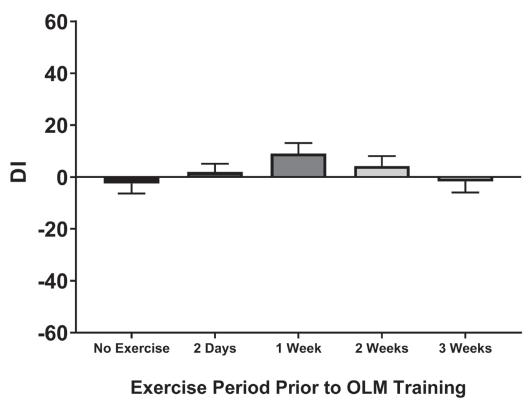

$\mathbf{F}$

Object Preference During OLM 24hr Test

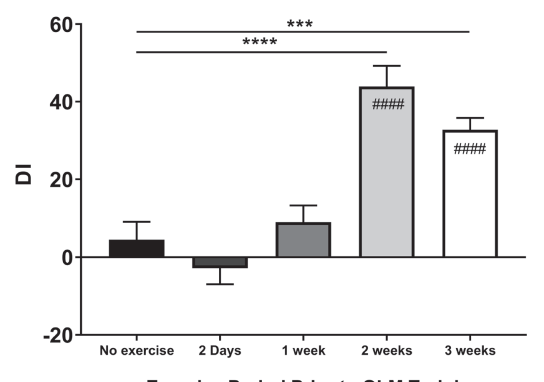

Exercise Period Prior to OLM Training

Figure 1. Effect of exercise on long-term object location memory. (A) Experimental schematic. Mice were given access to a running wheel for $2 \mathrm{~d}, 1,2$, or $3 \mathrm{wk}$ prior to object location memory (OLM) behavioral training and testing. OLM testing was performed $24 \mathrm{~h}$ after training, and mice were returned to standard home cages overnight. Mice were habituated to the OLM chamber for $6 \mathrm{~d}$ prior to OLM training regardless of exercise regime. A sedentary group of mice was also included that had no access to running wheels. (B) The mean total distance run by mice in each experimental group. (C): Mean time spent attending to objects during OLM training. There was no difference between the groups. $(D)$ Mean discrimination index for each group during OLM training. Mice showed no preference for objects during training. (E) Mean time spent attending to objects during OLM testing. There was no difference between the groups. $(F)$ Mean discrimination index for each group during OLM testing. Mice that had no running wheel access showed no object location memory at $24 \mathrm{~h}$. Mice that had run for the No exercise control group. Significant between-group comparisons are denoted by ${ }^{* * *} / * * * *$. Significant comparisons between Test discrimination index (DI) and Training DI are denoted by \#\#\#\#. All data shown is mean \pm SEM. $\left.{ }^{(* \star}\right) P<0.001,\left({ }^{* \star \star} / \# \# \# \#\right) P<0.0001$.

object preference during training. Following training, mice were returned to standard home cages for $24 \mathrm{~h}$. Mice were then analyzed for long-term OLM, by placing them back in the OLM chambers, where one object had been moved to a novel location. The total time spent investigating both objects was not significantly different between groups (Fig. $1 \mathrm{E}, F_{(4,45)}=1.343, P=0.2688$ ), suggesting no differences in object exploration. However, OLM DI was shown to be affected by the previous exercise paradigm (Fig. 1F; $F_{(4,45)}=$ $18.04, P<0.0001)$. Mice that received no exercise demonstrated a DI near 0 , indicating no object preference and thus no object 
location memory. Similarly, mice that received running wheel access for $2 \mathrm{~d}$, or $1 \mathrm{wk}$ showed no significant difference in OLM DI compared to sedentary controls ( 2 d vs. Sedentary: $P=0.7995$; 1 wk vs. Sedentary: $P>0.9999)$. However, mice that received either 2- or 3-wk of exercise prior to OLM training displayed a significant novel object location preference during testing, as shown by significantly increased DI, suggesting an enhanced object location memory compared to both the Sedentary control group, and to the corresponding OLM Training DI $(2$ wk vs. Sedentary: $P<$ 0.0001 ; 3 wk vs. Sedentary: $P=0.0002$; Comparisons of OLM Test DI to Training DI: Sedentary: $P=0.9473 ; 2$ d: $P>0.9999$; 1 wk: $P>0.9999 ; 2$ wk: $P<0.0001 ; 3$ wk: $P<0.0001)$. Thus, $2-$ and 3 -wk of running wheel access provides sufficient exercise to enable long-term OLM following a training paradigm that is subthreshold for sedentary controls, whereas $2 \mathrm{~d}$ or $1 \mathrm{wk}$ of running wheel access does not.

\section{Effects of a return to sedentary behavior and reactivation exercise on OLM performance in mice that have experienced $2 \mathrm{wk}$ of running}

We next examined the effect of a return to sedentary activity prior to OLM training (Fig. 2). A separate cohort of mice received a $2 \mathrm{wk}$ period of running wheel access, before being returned to sedentary home cages for either 3, 7, or $14 \mathrm{~d}$. Standard subthreshold OLM training and testing was then performed (Fig. 2A). An additional cohort was provided running wheel access for $2 \mathrm{wk}$, followed by a sedentary delay period of 3,7 , or $14 \mathrm{~d}$, but then also given an additional $2 \mathrm{~d}$ "reactivating" period of exercise, to determine if the effects of the sedentary delay could be rescued by the addition of a short period of exercise. OLM handling and habituation were performed on each mouse identically as above, regardless of cage type (Fig. 2A).

The mean total cumulative exercise recorded from each group during the initial 2 wk period was not significantly different (Fig. $\left.2 \mathrm{~B} ; F_{(5,52)}=2.269, P=0.0611\right)$, demonstrating that each group received a similar quantity of exercise during the $2 \mathrm{wk}$ period ( $2 \mathrm{wk}$ exercise, +3 d sedentary: $M=84,341 \mathrm{~m}, S D=25,839 \mathrm{~m} ; 2 \mathrm{wk} 3 \mathrm{~d}+$ 2 d: $M=97,633 \mathrm{~m}, S D=19,999 \mathrm{~m} ; 2$ wk7 d: $M=98,063 \mathrm{~m}, S D=$ 30,902 m; 2 wk7 d +2 d: $M=111,903 \mathrm{~m}, S D=24,391 \mathrm{~m} ; 2$ wk14 d: $M=112,315 \mathrm{~m}, S D=22,032 \mathrm{~m} ; 2$ wk14 d+2 d: $M=111,498 \mathrm{~m}$, $S D=20,152 \mathrm{~m}$ ). Exercise data from mice that had $2 \mathrm{wk}$ of running wheel access previously presented in Figure $1 \mathrm{~B}$ is shown again for comparison (Fig. 2B). The mean cumulative exercise recorded from each group that underwent $2 \mathrm{~d}$ of reactivation exercise was also not significantly different (Fig. $2 \mathrm{C} ; F_{(3,29)}=2.393, P=0.0888$ ).

Following initial exercise, sedentary period, and reactivating exercise, mice from each group received OLM training. Each group of mice displayed a similar total object exploration time during training, indicating no effect of exercise treatment on object exploration (Fig. 2D; Two-way ANOVA with a Group (4) $\times$ Reactivation (2) design: Effect of Group: $F_{(3,61)}=1.941, P=0.132$; effect of Reactivation: $F_{(1,61)}=4.235, P=0.044$; Interaction: $F_{(2,61)}=0.175$, $P=0.840$; Bonferroni post-hoc tests comparing No Reactivation to Reactivation within each group: 2 wk3 d: $P=0.5175 ; 2$ wk7 d: $P=0.4097 ; 2$ wk14 d: $P>0.9999)$. There was also no significant difference in DI for the objects, indicating no object preference during OLM training (Fig. 2E; Effect of Group: $F_{(3,61)}=0.833, P=0.481$; effect of Reactivation: $F_{(1,61)}=0.585, P=0.447$; interaction: $F_{(2,61)}=$ $0.062, P=0.940)$.

Following training, mice were returned to standard home cages for $24 \mathrm{~h}$, and then analyzed for long-term OLM as stated above. The total time spent investigating objects during testing was not significantly different between groups (Fig. 2F; Effect of Group: $F_{(3,63)}=1.677, P=0.181$, effect of Reactivation: $F_{(1,63)}=$ $0.290, P=0.592$; interaction: $\left.F_{(2,63)}=0.812, P=0.449\right)$, while the
DI was shown to be affected by the given exercise paradigm (Fig. 2G; Effect of Group: $F_{(3,63)}=7.853, P<0.0001$; Effect of Reactivation: $F_{(1,63)}=5.079, P=0.028$; interaction: $F_{(2,63)}=0.192$, $P=0.826$ ). A 3 -d sedentary period within a standard home cage was sufficient to significantly decrease OLM performance compared to mice with 2 -wk of running with no sedentary period (Fig. 2G; comparing $2 \mathrm{wk}$ DI to $2 \mathrm{wk} 3 \mathrm{~d}$ DI: $P=0.0268$ ). Similarly, sedentary delay periods of 7- and 14-d also counteracted the beneficial effect on subthreshold OLM performance from 2-wk of running (Fig. 2G; 2 wk vs. 2 wk7 d: $P=0.0225 ; 2$ wk vs. 2 wk14 d: $P=0.0008)$. However, exercise-induced OLM enhancement was retained in those mice that received $2 \mathrm{~d}$ of reactivating exercise following either 3- or 7-d sedentary delay, but not 14-d sedentary delay (Fig. $2 \mathrm{G} ; 2$ wk vs. 2 wk3 d $+2 \mathrm{~d}: P>0.9999 ; 2$ wk vs. 2 wk7 $\mathrm{d}+2 \mathrm{~d}: P=0.3156 ; 2$ wk vs. 2 wk $14 \mathrm{~d}+2 \mathrm{~d}: P=0.0077$; comparing within groups No reactivation vs. $+2 \mathrm{~d}$ Reactivation: 2 wk3 d: $P=$ 0.5832; 2 wk7 d: $P>0.9999 ; 2$ wk14 d: $P>0.9999$; comparing OLM DI during Training to Test: 2 wk: $P<0.0001 ; 2$ wk3 d: $P=$ 0.0836; 2 wk3 d +2 d: $P=0.0004 ; 2$ wk7 d: $P=0.2774 ; 2$ wk7 d +2 d: $P=0.0023 ; 2$ wk14 d: $P>0.9999 ; 2$ wk14 d+2 d: $P>0.9999)$. These data suggest that exercise-induced OLM enhancement decays following a return to sedentary activity, but can be "reactivated" by a short period of exercise.

\section{Increasing initial exercise period length does not increase the sedentary period that can be rescued by $2 \mathrm{~d}$ exercise}

Given that $2 \mathrm{~d}$ of reactivating exercise could maintain exerciseenhanced OLM after 3 and 7, but not $14 \mathrm{~d}$, we next investigated whether extending the length of the initial exercise period could increase the length of sedentary delay that could still be rescued by $2 \mathrm{~d}$ of reactivating exercise (Fig. 3). Mice were given access to running wheels for a period of 3-wk, followed by a delay period of either 7- or 14-d, prior to OLM training and testing. A separate cohort of mice was again reexposed to the running wheels for 2-d just prior to OLM training and testing (Fig. 3A).

Exercise during the 3-wk initial exercise period was recorded for each mouse. The mean total distance run by each group of mice during the $3 \mathrm{wk}$ period was not significantly different (Fig. 3B: $\left.F_{(2,29)}=1.591, P=0.2130\right)$, suggesting each group received a similar quantity of exercise. Exercise data from mice that had 3 wk of running wheel access previously presented in Figure $1 \mathrm{~B}$ is shown again for comparison (Fig. 3B). The mean cumulative exercise recorded from each group that underwent an additional $2 \mathrm{~d}$ of reactivation exercise was also not significantly different (Fig. 3C; $\left.F_{(2,22)}=2.766, P=0.0848\right)$.

Following the initial 3-wk exercise period, sedentary period, and reactivating exercise period, mice from each group then received OLM training. Each group of mice displayed a similar total object exploration time during training, indicating no effect of exercise treatment on object exploration (Fig. 3D: Two-way ANOVA with a Group (2) $\times$ Reactivation (2) design: Effect of Group: $F_{(2,37)}$ $=1.705, P=0.196$; effect of Reactivation: $F_{(1,37)}<0.001, P=0.998$; Interaction: $\left.F_{(1,37)}=2.847, P=0.100\right)$. There was also no significant difference in DI for the objects, indicating no object preference during training (Fig. 3E: Effect of Group: $F_{(2,37)}=0.032, P=0.969$; effect of Reactivation: $F_{(1,37)}=0.088, P=0.768$; interaction: $F_{(1,37)}$ $=0.006, P=0.940$ ). Following training, mice were returned to standard home cages for $24 \mathrm{~h}$, and then analyzed for long-term OLM as described above. The total time spent investigating objects during testing was not significantly different between groups (Fig. 3F; Effect of Group: $F_{(2,37)}=2.895, P=0.068$, effect of Reactivation: $F_{(1,37)}=0.203, P=0.655$; interaction: $\left.F_{(1,37)}=0.142, P=0.709\right)$.

Exercise-induced OLM enhancement significantly decreased in all groups, except for those mice that received $2 \mathrm{~d}$ of reactivating 
A

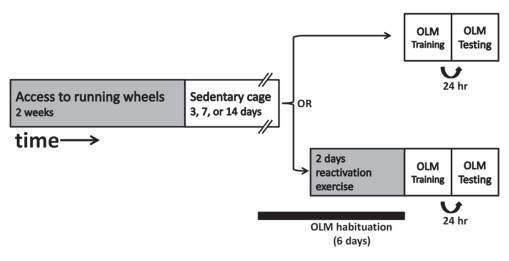

B

Distance Run During 2 Week Exercise Period

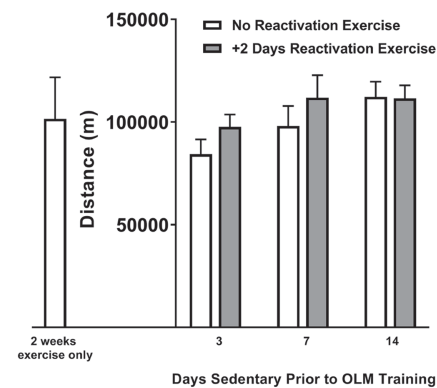

C

Distance Run During

2 Day Reactivation Exercise

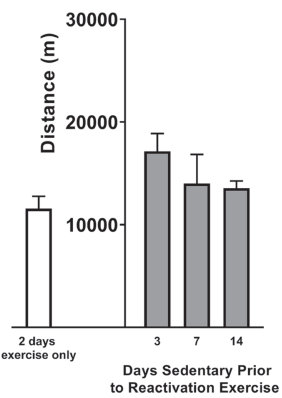

D Total Object Exploration Time During OLM Training

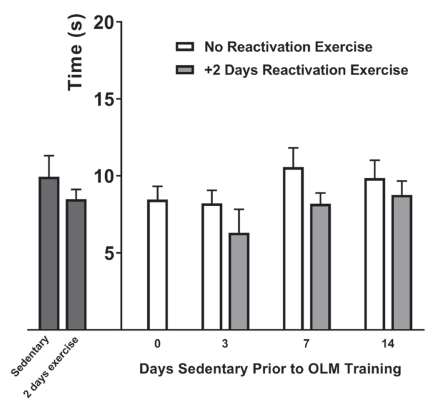

F Total Object Exploration Time During OLM 24hr Test

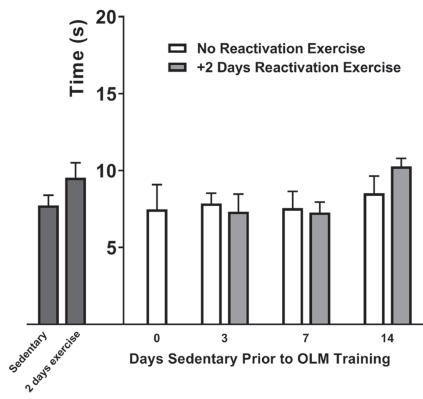

E Object Preference During OLM Training

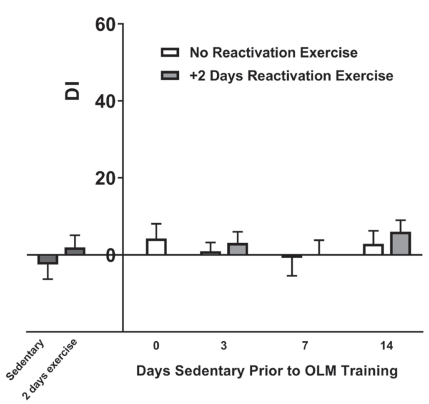

G Object Preference During OLM 24hr Test

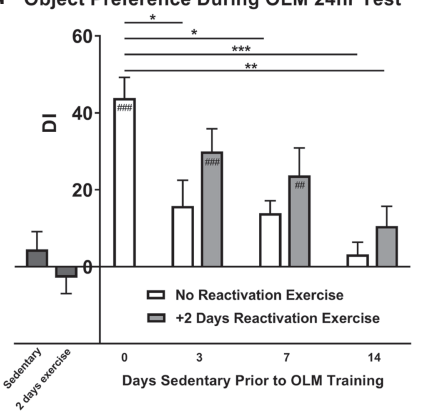

Figure 2. Effect of a sedentary period and reactivating exercise on 2 wk exercise-enhanced long-term object location memory. $(A)$ Experimental schematic. Mice were given access to a running wheel for $2 \mathrm{wk}$. Mice were then split into groups and returned to their home cages for either 3, 7, or $14 \mathrm{~d}$. Mice were then either immediately trained and tested for OLM, or given access to running wheels for another $2 \mathrm{~d}$ (reactivation exercise) prior to OLM training and testing. OLM testing was performed $24 \mathrm{~h}$ after training. All mice were habituated to the OLM chamber for the $6 \mathrm{~d}$ prior to OLM training. (B) The mean total distance run by mice in each experimental group during the $2 \mathrm{wk}$ exercise period. There was no significant difference in the amount of exercise each group received during this period. Distance data from 2 wk exercise mice presented in Figure 1B also shown for comparison. (C) The mean total distance run by mice that underwent $2 \mathrm{~d}$ reactivation exercise. There was no significant difference in the amount of exercise each group received during this period. Distance data from Figure 1B also shown for comparison. (D) Mean time spent attending to objects during OLM training. Data from Sedentary and $2 \mathrm{~d}$ exercise controls presented in Figure 1C are also shown for comparison. There was no difference between the groups. (E) Mean DI for each group during OLM training. Data from Sedentary and $2 \mathrm{~d}$ exercise controls presented in Figure 1D are also shown for comparison. Mice showed no preference for objects during training. $(F)$ Mean time spent attending to objects during OLM testing. Data from Sedentary and $2 \mathrm{~d}$ exercise controls presented in Figure $1 \mathrm{E}$ are also shown for comparison. There was no difference between the groups. (G) Mean DI for each group during OLM testing. Data from Sedentary and $2 \mathrm{~d}$ exercise controls presented in Figure $1 \mathrm{~F}$ are also shown for comparison. Mice that run for $2 \mathrm{wk}$ followed by either a 3, 7, or $14 \mathrm{~d}$ sedentary period show a significant decrease in preference for novel object location, compared to mice that run for $2 \mathrm{wk}$ with no sedentary period. Mice that then receive a $2 \mathrm{~d}$ period of reactivation exercise following either 3 or 7 sedentary days show a significant increase in novel object preference during testing compared to training. However, $2 \mathrm{~d}$ of reactivation exercise is not sufficient to significantly improve object location memory in mice that have had a sedentary delay period of $14 \mathrm{~d}$. All data shown is mean \pm SEM. Between-groups comparisons denoted by ${ }^{* \prime} s$, within-group comparisons denoted by \#'s. $\left({ }^{*}\right) P<0.05$, $\left({ }^{* *} / \# \#\right) P<0.01$, $\left.{ }^{(* \star} / \# \# \#\right) P<0.001$.

exercise following a 7-d sedentary delay (Fig. 3G; Effect of Group: $F_{(2,37)}=22.752, P<0.0001$; Effect of Reactivation: $F_{(1,37)}=10.327$, $P=0.003$; interaction: $F_{(1,37)}=3.422, P=0.072$; post-hoc tests comparing each group to 3 wk DI: 3 wk $7 \mathrm{~d}: P=0.0016 ; 3 \mathrm{wk} 7 \mathrm{~d}+2: P>$ 0.9999; 3 wk 14 d: $P<0.0001 ; 3$ wk $14 \mathrm{~d}+2$ d: $P=0.0005$; comparing within groups No Reactivation vs. $+2 \mathrm{~d}$ Reactivation: $3 \mathrm{wk}$ 7 d: $P=0.0068 ; 3$ wk 14 d: $P>0.9999$; comparing OLM DI during
Training to Test: 3 wk: $P<0.0001 ; 3$ wk $7 \mathrm{~d}: P=0.5285 ; 3$ wk $7 \mathrm{~d}+$ 2 d: $P<0.0001 ; 3$ wk $14 \mathrm{~d}: P>0.9999 ; 3$ wk $14 \mathrm{~d}+2 \mathrm{~d}: P=0.9212)$. These data suggest that increasing the length of the initial period of exercise has no effect on the decay of exercise-induced memory enhancement caused by a return to sedentary activity, but may increase the ability for reactivation exercise to enhance cognition after a 7-d delay. 
A

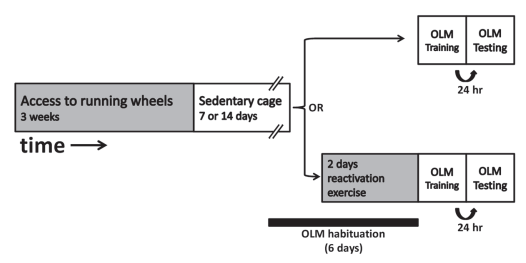

B

Distance Run During 3 Week Exercise Period

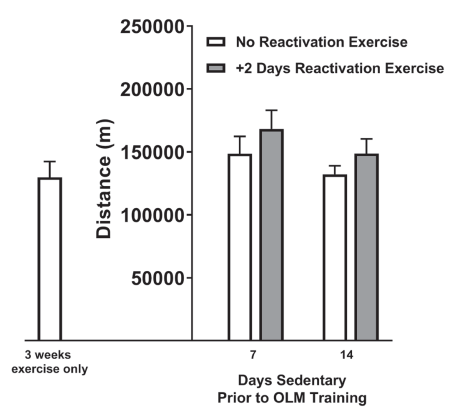

C Distance Run During 2 Day Reactivation Exercise

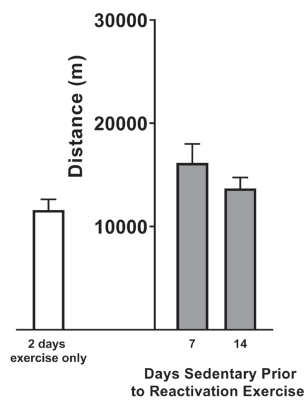

D Total Object Exploration Time During OLM Training

E Object Preference During OLM Training
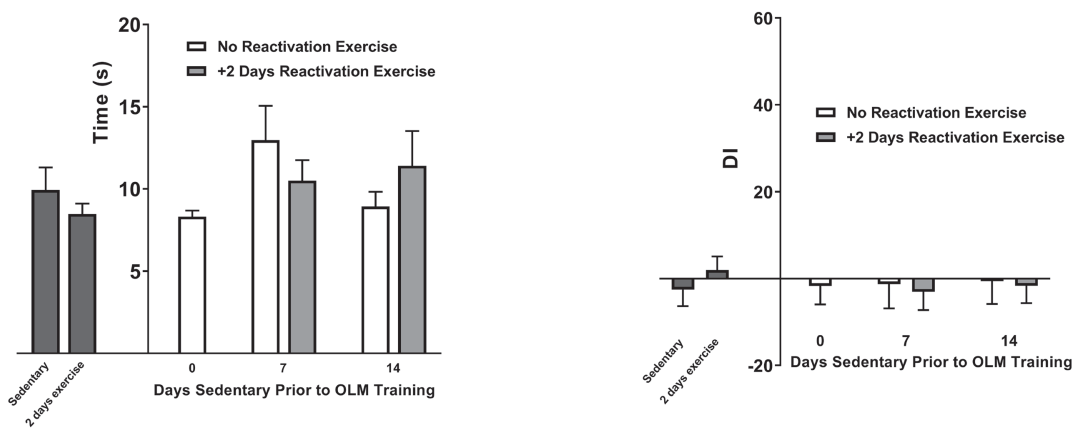

F Total Object Exploration Time During OLM 24hr Test

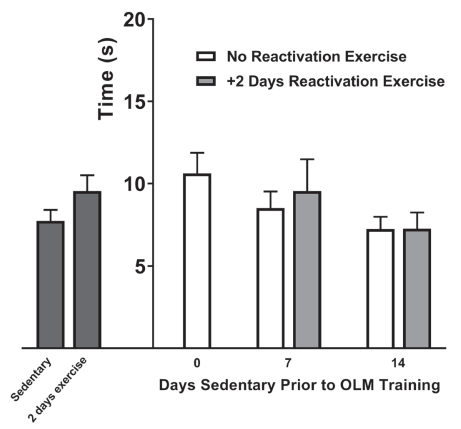

G Object Preference During OLM 24hr Test

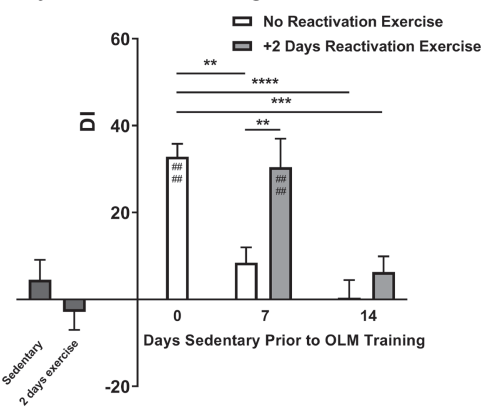

Figure 3. Effect of sedentary activity and reactivating exercise on 3 wk exercise-enhanced long-term object location memory. $(A)$ Experimental schematic. Mice were given access to a running wheel for $3 \mathrm{wk}$. Mice were then split into groups and were returned to their home cages for either 7 or $14 \mathrm{~d}$. Mice were then either immediately trained and tested for object location memory (OLM), or given access to running wheels for another $2 \mathrm{~d}$ (reactivation exercise) prior to OLM training and testing. OLM testing was performed $24 \mathrm{~h}$ after training. All mice were habituated to the OLM chamber for the $6 \mathrm{~d}$ prior to OLM training. $(B)$ The mean total distance run by mice in each experimental group during the 3 wk exercise period. There was no significant difference in the amount of exercise each group received during this period. Distance data from 3 wk exercise mice presented in Figure $1 \mathrm{~B}$ also shown for comparison. (C) The mean total distance run by mice that underwent $2 \mathrm{~d}$ reactivation exercise. There was no significant difference in the amount of exercise each group received during this period. Distance data from Figure $1 \mathrm{~B}$ also shown for comparison. $(D)$ Mean time spent attending to objects during OLM training. Data from Sedentary and $2 \mathrm{~d}$ exercise controls presented in Figure $1 \mathrm{C}$ are also shown for comparison. There was no difference between the groups. (E) Mean DI for each group during OLM training. Data from Sedentary and $2 \mathrm{~d}$ exercise controls presented in Figure 1D are also shown for comparison. Mice showed no preference for objects during training. $(F)$ Mean time spent attending to objects during OLM testing. Data from Sedentary and $2 \mathrm{~d}$ exercise controls presented in Figure 1E are also shown for comparison. There was no difference between the groups. (G) Mean DI for each group during OLM testing. Data from Sedentary and $2 \mathrm{~d}$ exercise controls presented in Figure 1F are also shown for comparison. Mice that run for 3 wk followed by either a 7- or 14-d sedentary period show a significant decrease in OLM performance compared to mice that run for 3 wk with no sedentary period. Mice that experience a 2-d period of reactivation exercise following the sedentary period show a significant increase in novel object preference during OLM testing compared to training. However, $2 \mathrm{~d}$ of reactivation exercise was not sufficient to significantly improve object location memory in mice that had a sedentary delay period of $14 \mathrm{~d}$. All data shown is mean \pm SEM. Between-groups comparisons denoted by ${ }^{* \prime}$, within-group comparisons denoted by $\#$ 's. (**) $P<0.01$, $\left.{ }^{* * *}\right) P<$ 0.001 , (****\#\#\#\#) $P<0.0001$.

\section{Discussion}

This study examined the effect of exercise period duration, sedentary period duration, and reactivating exercise on cognition in mice, using a specific subthreshold object location memory (OLM) training paradigm. Mice with no running wheel access were unable to distinguish between novel and familiar object locations $24 \mathrm{~h}$ after training, as were mice with 2-d, or 1-wk of running wheel access. However, 2- or 3-wk of running wheel access enabled the formation of long-term object location memories, using the same OLM training paradigm. The effect of a return to sedentary activity on subthreshold OLM was then examined; the 
exercise-induced OLM enhancement seen in mice that had 2-wk of running wheel access was shown to decay to sedentary control levels after three sedentary days. However, OLM could be reenhanced in these mice by an additional period of running wheel access for $2 \mathrm{~d}$, which by itself was insufficient to enable a long-term OLM to form. These data may suggest a "molecular memory" for the experience of exercise, in that once exercise reaches a certain threshold, both cognition, and the ability of future exercise to improve cognition, are enhanced. These findings provide new information regarding the time course of cognitive enhancement by exercise, and may have implications for the recommendations of minimum exercise required for cognitive enhancement in humans.

\section{Exercise enables memory encoding from subthreshold training}

The demonstration of exercise enhancing cognition in rodents is in line with numerous other studies using a variety of behavioral tests, including radial arm maze, Morris water maze, Y-maze, context fear conditioning, and novel object recognition (Fordyce and Farrar 1991; Anderson et al. 2000; Baruch et al. 2004; Van der Borght et al. 2007; Fahey et al. 2008). Importantly, we replicate the results of Intlekofer et al. (2013) demonstrating that exercise enables the encoding of long-term OLM that is not typically encoded in nonexercising mice. While it was previously shown that using the subthreshold training paradigm, 3-wk of running wheel access enabled OLM to form, here we extend these findings, demonstrating that 2 -wk of exercise is also sufficient, while 1 wk or $2 \mathrm{~d}$ of running wheel access is not. These data suggest an exercise threshold for cognitive improvement in the subthreshold OLM task, below which there is insufficient signaling for the neurobiological mechanisms that underlie cognitive enhancement to occur. Our previous findings implicated hippocampal BDNF protein levels as a molecular mechanism required for exercise-induced OLM enhancement. Hippocampal BDNF is an important mediator for the formation of hippocampal-dependent memory (Mu et al. 1999; Bekinschtein et al. 2007; Heldt et al. 2007), and BDNF protein levels progressively increase with longer running duration (Adlard et al. 2004; Berchtold et al. 2005). Indeed, we have demonstrated a direct relationship between hippocampal BDNF levels and performance in the radial arm water maze (Berchtold et al. 2010), and shown that hippocampal BDNF is required for OLM following subthreshold training (Intlekofer et al. 2013). It is thus reasonable to suggest that the exercise-induced cognitive enhancement seen in the present study is mediated via a BDNF mechanism.

\section{Loss of memory-enhancement upon return to sedentary activity}

Mice that experienced a 3- or 7-d sedentary delay period between running wheel access and OLM testing displayed a significant decrease in OLM performance compared to nonsedentary delayed controls. This decay in exercise-induced memory enhancement is more rapid than that seen in other studies (Radak et al. 2006; Berchtold et al. 2010), which may reflect an increase in the difficulty of the behavioral paradigm used. Furthermore, increasing the initial exercise period from 2 - to 3 -wk did not result in a noticeable increase in OLM after a 7-d sedentary delay. These data suggest that the sedentary decay of exercise-induced OLM enhancement is not affected by the quantity of initial physical activity experienced.

\section{Reintroduction to physical activity reactivates a "memory" for exercise-induced cognitive enhancement}

On its own, $2 \mathrm{~d}$ of running wheel access had no effect on OLM compared to sedentary controls. However, mice that had lost the exercise-induced OLM enhancement due to a return to sedentary housing, displayed a recovery in their ability to form OLM when reexposed to 2 -d of running wheel access. This reactivation effect also displayed a time course of decay; reactivating exercise was successful following three- or seven-sedentary days, while 14-sedentary days prior to the reactivation period did not result in significantly improved OLM when compared to training. These data are broadly in accordance with our previous work demonstrating a "molecular memory" for BDNF induction by exercise, which further implicates hippocampal BDNF levels as a molecular mechanism underlying the cognitive enhancement seen in the present study (Berchtold et al. 2005; Intlekofer et al. 2013). Hippocampal BDNF protein returns to sedentary levels after 7-14 sedentary days, but is rapidly reexpressed in response to $2 \mathrm{~d}$ of running wheel access (Berchtold et al. 2005). While the time course of BDNF expression in the hippocampus differs slightly compared to the cognitive enhancement shown here, this may be explained by the use of rats compared to mice, as well as the difficulty of the behavioral paradigm used, as previously discussed.

While transient changes in hippocampal BDNF levels may explain cognitive enhancement by exercise or the return to sedentary-type cognition following a return to sedentary housing, the molecular priming for BDNF induction by exercise requires further investigation. There is some evidence that such molecular priming may occur via an epigenetic mechanism. Epigenetic modifications have been shown to modulate learning and memory outcomes (Levenson and Sweatt 2005; Sleiman et al. 2016; Campbell and Wood 2019). Both exercise and sodium butyrate (NaB; a histone deacetylase (HDAC) inhibitor) treatment have been shown to induce expression of the same BDNF transcripts in the hippocampus, and to improve OLM (Stefanko et al. 2009; Intlekofer et al. 2013). Exercise and $\mathrm{NaB}$ are also shown to increase acetylation of histone $\mathrm{H} 4$ at promoters for the same $b d n f$ gene transcripts (Intlekofer et al. 2013). Furthermore, exercise has been shown to induce hippocampal accumulation of the endogenous ketone body D- $\beta$-hydroxybutyrate, which can also inhibit class I HDACs (Sleiman et al. 2016). Hippocampal neurons treated with D- $\beta$-hydroxybutyrate in vitro show increased acetyl-histone H3, decreased HDAC2 and HDAC3 occupancy at the bdnf promoter, and increased BDNF expression (Sleiman et al. 2016). These data suggest that exercise may induce a permissive chromatin state that allows the rapid expression of cognitive-enhancing genes such as $b d n f$ following further exercise stimulus. Future studies may thus examine other genes affected by epigenetic changes that occur following periods of exercise and sedentary activity that are similar to the exercise regimens presented in the current study.

\section{Clinical implications of findings}

If the data presented in the current study is applicable to humans, our results suggest that while cognition is enhanced by exercise, it can also deteriorate from a sedentary lifestyle. However, a regular exercise routine whereby physical activity is experienced only occasionally may be sufficient to preserve normal cognitive function.

\section{Materials and Methods}

\section{Animals}

All experiments were conducted in accordance with the National Institutes of Health guidelines for animal care and use, and were approved by the Institutional Animal Care and Use Committee of the University of California at Irvine. Male, 6-wk-old C57Bl/6J mice (Jackson Laboratory) were individually housed with food and water ad libitum, and allowed $1 \mathrm{wk}$ acclimation to the vivari$\mathrm{um}$ prior to experiments. Lights were maintained on a $12 \mathrm{~h}$ 
light-dark cycle, with all behavior performed during the light phase. Total number of mice analyzed: 182 .

\section{Exercise treatment}

Mice were split into separate groups and spent different periods of time in either exercise cages (housed with a running wheel) or sedentary cages (standard cages). Exercise cages consisted of 24 $\times 35 \times 20 \mathrm{~cm}$ clear plastic, containing a running wheel $40 \mathrm{~cm}$ in circumference, $12.7 \mathrm{~cm}$ diameter (Lafayette). Voluntary running was monitored via a computer system (Lafayette) with activity recorded every $6 \mathrm{~h}$ ( 4 times per day). Exercise regimes consisted of an initial period of exercise $(0,1,2$, or $3 \mathrm{wk})$, followed by a sedentary period during which mice were returned to home cages $(0$, 3,7 , or $14 \mathrm{~d}$ ). Some groups of mice then received a period of reactivating exercise, consisting of $2 \mathrm{~d}$ access to the running wheels (see Figs. 1A, 2A, 3A).

\section{Subthreshold object location memory (OLM) testing}

OLM training and testing was performed as described by Vogel-Ciernia and Wood (2014). Briefly, OLM chambers consisted of $30 \times 30 \times 40 \mathrm{~cm}$ boxes, containing $1 \mathrm{~cm}$ deep corn cob bedding. Nine days prior to OLM training, each mouse was handled for 2 min per day, for 5 consecutive days, within the OLM training room. Each day for $6 \mathrm{~d}$ prior to training, each mouse was habituated to the OLM chambers for 10 min per day. Mouse handling and habituation thus overlapped for $2 \mathrm{~d}$. Regardless of the exercise regime, each mouse was returned to the standard sedentary home cage the night before OLM training. On OLM training day, two $100 \mathrm{~mL}$ beakers filled with cement were placed into each OLM chamber upside down. Subthreshold OLM training consisted of allowing each mouse to explore the chamber and objects for $3 \mathrm{~min}$, before returning to home cages. Twenty-four hours after training, OLM testing was performed, consisting of moving one of the beakers and allowing each mouse to explore the chamber and objects for $5 \mathrm{~min}$. Exploratory behavior was recorded and scored manually offline using BORIS v7 software (Friard and Gamba 2016). Object exploration was defined as the interaction time when the mouse's nose was within $1 \mathrm{~cm}$ of the object and was pointing directly at the object, discounting when the mouse was on top of the object, looking over the object, or engaged in repetitive behavior such as digging at the base of the object (Vogel-Ciernia and Wood 2014). Object exploration time was recorded and used to calculate a DI = ((Novel object location exploration time - Familiar object location exploration time)/Total object exploration time $\times 100)$. Typically, a DI $>20$ indicates a robust OLM (Vogel-Ciernia and Wood 2014). Mice that did not have a total object exploration time of at least 3 sec during either training or testing were excluded. Additionally, mice that displayed an object preference during training as evidenced by a DI of \pm 20 were also excluded. OLM training and tests were performed with knowledge of the previous exercise regime of each mouse, while manual offline OLM scoring was blinded. Total mice excluded from study: 18 .

\section{Statistics}

One-way ANOVAs were used to analyze total distances run (Figs. $1 \mathrm{~B}, 2 \mathrm{~B}, \mathrm{C}, 3 \mathrm{~B}, \mathrm{C})$, and the effect of exercise on OLM Acquisition Exploration Time, Acquisition DI, Testing Exploration Time, and Testing DI (Fig. 1C-F). For OLM data, Bonferroni post-hoc tests were performed comparing each exercise group to the sedentary control group following significant ANOVA results. For OLM data following either 2- or 3-wk of exercise with a delay period and reactivation exercise, two-way ANOVAs were performed, with a Delay group $\times$ Reactivation design (Figs. $2 D-G, 3 D-G$ ). Significant effects were examined using Bonferroni post-hoc tests comparing each group to the relevant exercise control. Training and Test DIs from each mouse were also compared following a repeated measures two-way ANOVA (Figs. 1F, 2G, 3G). Data was analyzed using GraphPad Prism software, and SPSS (IBM).

\section{Acknowledgments}

This work was made possible by National Institute on Aging (NIA), United States (R01AG057558, R01AG051807).

\section{References}

Adlard PA, Perreau VM, Engesser-Cesar C, Cotman CW. 2004. The timecourse of induction of brain-derived neurotrophic factor mRNA and protein in the rat hippocampus following voluntary exercise. Neurosci Lett 363: 43-48. doi:10.1016/j.neulet.2004.03.058

Alderman BL, Olson RL. 2018. Prescribing exercise for mental health. In The exercise effect on mental health-neurobiological mechanisms (ed. Budde $\mathrm{H}$, Wegner M), pp. 411-441. CRC Press, Boca Raton.

Anderson BJ, Rapp DN, Baek DH, McCloskey DP, Coburn-Litvak PS, Robinson JK. 2000. Exercise influences spatial learning in the radial arm maze. Physiol Behav 70: 425-429. doi:10.1016/S0031-9384(00) 00282-1

Baruch DE, Swain RA, Helmstetter FJ. 2004. Effects of exercise on Pavlovian fear conditioning. Behav Neurosci 118: 1123-1127. doi:10.1037/ 0735-7044.118.5.1123

Bekinschtein P, Cammarota M, Igaz LM, Bevilaqua LR, Izquierdo I, Medina JH. 2007. Persistence of long-term memory storage requires a late protein synthesis- and BDNF- dependent phase in the hippocampus. Neuron 53: 261-277. doi:10.1016/j.neuron.2006.11.025

Berchtold NC, Chinn G, Chou M, Kesslak JP, Cotman CW. 2005. Exercise primes a molecular memory for brain-derived neurotrophic factor protein induction in the rat hippocampus. Neuroscience 133: 853861. doi:10.1016/j.neuroscience.2005.03.026

Berchtold NC, Castello N, Cotman CW. 2010. Exercise and time-dependent benefits to learning and memory. Neuroscience 167: 588-597. doi:10 .1016/j.neuroscience.2010.02.050

Campbell RR, Wood MA. 2019. How the epigenome integrates information and reshapes the synapse. Nat Rev Neurosci 20: 133-147. doi:10.1038/ s41583-019-0121-9

Cotman CW, Berchtold NC. 2002. Exercise: a behavioral intervention to enhance brain health and plasticity. Trends Neurosci 25: 295-301. doi:10 .1016/S0166-2236(02)02143-4

Fahey B, Barlow S, Day JS, O'Mara SM. 2008. Interferon- $\alpha$-induced deficits in novel object recognition are rescued by chronic exercise. Physiol Behav 95: 125-129. doi:10.1016/j.physbeh.2008.05.008

Fordyce DE, Farrar RP. 1991. Enhancement of spatial learning in F344 rats by physical activity and related learning-associated alterations in hippocampal and cortical cholinergic functioning. Behav Brain Res 46: 123-133. doi:10.1016/S0166-4328(05)80105-6

Friard O, Gamba M. 2016. BORIS: a free, versatile open-source event-logging software for video/audio coding and live observations. Methods Ecol Evol 7: 1325-1330.

Gomez-Pinilla F, Hillman C. 2013. The influence of exercise on cognitive abilities. Compr Physiol 3: 403-428. doi:10.1002/cphy.c110063

Haskell WL, Lee IM, Pate RR, Powell KE, Blair SN, Franklin BA, Macera CA, Heath GW, Thompson PD, Bauman A. 2007. Physical activity and public health: updated recommendation for adults from the American College of Sports Medicine and the American Heart Association. Med Sci Sports Exerc 39: 1423-1434. doi:10.1249/mss.0b013e3180616b27

Heldt SA, Stanek L, Chhatwal JP, Ressler KJ. 2007. Hippocampus-specific deletion of BDNF in adult mice impairs spatial memory and extinction of aversive memories. Mol Psychiatry 12: 656-670. doi:10.1038/sj.mp .4001957

Intlekofer KA, Berchtold NC, Malvaez M, Carlos AJ, McQuown SC, Cunningham MJ, Wood MA, Cotman CW. 2013. Exercise and sodium butyrate transform a subthreshold learning event into long-term memory via a brain-derived neurotrophic factor-dependent mechanism. Neuropsychopharmacology 38: 2027-2034. doi:10.1038/npp .2013 .104

Lee IM, Skerrett PJ. 2001. Physical activity and all-cause mortality: what is the dose-response relation? Med Sci Sports Exerc 33: S459-S471. discussion S493-454. doi:10.1097/00005768-200106001-00016

Levenson JM, Sweatt JD. 2005. Epigenetic mechanisms in memory formation. Nat Rev Neurosci 6: 108-118. doi:10.1038/nrn1604

McQuown SC, Barrett RM, Matheos DP, Post RJ, Rogge GA, Alenghat T, Mullican SE, Jones S, Rusche JR, Lazar MA, et al. 2011. HDAC3 is a critical negative regulator of long-term memory formation. J Neurosci 31: 764774. doi:10.1523/JNEUROSCI.5052-10.2011

Mu JS, Li WP, Yao ZB, Zhou XF. 1999. Deprivation of endogenous brain-derived neurotrophic factor results in impairment of spatial learning and memory in adult rats. Brain Res 835: 259-265. doi:10.1016/ S0006-8993(99)01592-9

Nagamatsu LS, Flicker L, Kramer AF, Voss MW, Erickson KI, Hsu CL, Liu-Ambrose T. 2014. Exercise is medicine, for the body and the brain. $\mathrm{Br}$ J Sports Med 48: 943-944. doi:10.1136/bjsports-2013-093224 
Neeper SA, Gómez-Pinilla F, Choi J, Cotman CW. 1996. Physical activity increases mRNA for brain-derived neurotrophic factor and nerve growth factor in rat brain. Brain Res 726: 49-56. doi:10.1016/0006-8993(96) 00273-9

Radak Z, Toldy A, Szabo Z, Siamilis S, Nyakas C, Silye G, Jakus J, Goto S. 2006. The effects of training and detraining on memory, neurotrophins and oxidative stress markers in rat brain. Neurochem Int 49: 387-392. doi:10 .1016/j.neuint.2006.02.004

Sleiman SF, Henry J, Al-Haddad R, El Hayek L, Abou Haidar E, Stringer T, Ulja D, Karuppagounder SS, Holson EB, Ratan RR, et al. 2016. Exercise promotes the expression of brain derived neurotrophic factor (BDNF) through the action of the ketone body $\beta$-hydroxybutyrate. Elife 5: e15092. doi:10.7554/eLife.15092

Stefanko DP, Barrett RM, Ly AR, Reolon GK, Wood MA. 2009. Modulation of long-term memory for object recognition via HDAC inhibition. Proc Natl Acad Sci 106: 9447-9452. doi:10.1073/pnas.0903964106

Van der Borght K, Havekes R, Bos T, Eggen BJ, Van der Zee EA. 2007. Exercise improves memory acquisition and retrieval in the Y-maze task: relationship with hippocampal neurogenesis. Behav Neurosci 121: 324334. doi:10.1037/0735-7044.121.2.324

Vivar C, Potter MC, van Praag H. 2013. All about running: synaptic plasticity, growth factors and adult hippocampal neurogenesis. Curr Top Behav Neurosci 15: 189-210. doi:10.1007/7854_2012_220

Vogel-Ciernia A, Wood MA. 2014. Examining object location and object recognition memory in mice. Curr Protoc Neurosci 69: 31.1-31.17. doi:10 $.1002 / 0471142301 . n s 0831$ s69

Warburton DE, Nicol CW, Bredin SS. 2006. Health benefits of physical activity: the evidence. CMAJ 174: $801-809$. doi: $10.1503 / \mathrm{cmaj}$ .051351

WHO. 2010. Global recommendations on physical activity for health. World Health Organization, Geneva.

Received July 9, 2019; accepted in revised form October 11, 2019. 


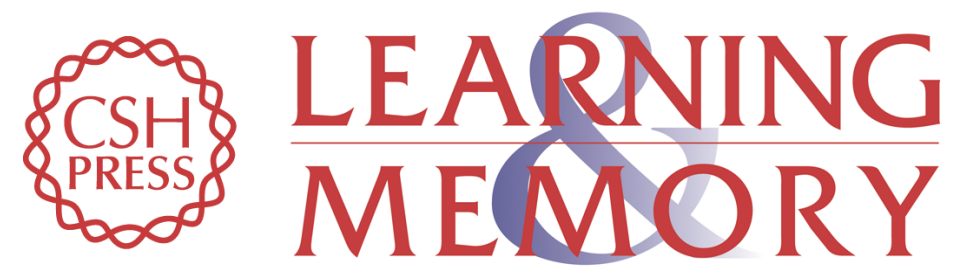

\title{
Exercise opens a temporal window for enhanced cognitive improvement from subsequent physical activity
}

\author{
Christopher W. Butler, Ashley A. Keiser, Janine L. Kwapis, et al.
}

Learn. Mem. 2019, 26:

Access the most recent version at doi:10.1101/Im.050278.119

\begin{aligned} & \hline References $\begin{array}{l}\text { This article cites } 28 \text { articles, } 4 \text { of which can be accessed free at: } \\ \text { http://learnmem.cshlp.org/content/26/12/485.full.html\#ref-list-1 }\end{array} \\ & \begin{aligned} \text { Creative } \\ \text { Commons } \\ \text { License }\end{aligned} \begin{array}{l}\text { This article is distributed exclusively by Cold Spring Harbor Laboratory Press for the } \\ \text { first } 12 \text { months after the full-issue publication date (see } \\ \text { http://learnmem.cshlp.org/site/misc/terms.xhtml). After } 12 \text { months, it is available under } \\ \text { a Creative Commons License (Attribution-NonCommercial } 4.0 \text { International), as } \\ \text { described at http://creativecommons.org/licenses/by-nc/4.0/. }\end{array} \\ & \begin{array}{c}\text { Receive free email alerts when new articles cite this article - sign up in the box at the } \\ \text { top right corner of the article or click here. }\end{array} \\ & \begin{array}{c}\text { Service } \\ \text { terting }\end{array}\end{aligned}$

